\section{Buchrezension zu: Methoden der Mikrobiologie}

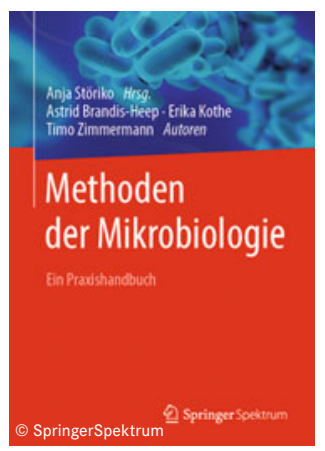

Methoden der Mikrobiologie Ein Praxishandbuch

Anja Störiko (Hrsg.)

Astrid Brandis-Heep, Erika

Kothe und Timo Zimmermann

294 S., 104 Abb., Springer Spektrum,

2020. $S C, 39,99 €$.

ISBN: 9783662605530

Auch als E-Book erhältlich

DOI: $10.1007 / \mathrm{s} 12268-020-1474-3$

(C) Der Autor 2020

Man sollte meinen, dass mikrobiologische Methoden aus klassischen Grundpraktika längst ausreichend literarisch unterfüttert wären. Tatsächlich aber fehlt eine solche Sammlung, die Hintergrundwissen mit direkt anwendbaren Laborprotokollen verbindet, im Bereich der mikrobiellen Physiologie fast gänzlich. Und genau diese Lücke schließt das vorliegende, von Anja Störiko herausgegebene Buch, in dem drei erfahrene Autor/inn/en ihr in Jahrzehnten mikrobiologischer Grundpraktika gewonnenes Wissen sowie vielfach erprobte Versuche gut lesbar und nachvollziehbar aufbereiten. Man merkt dem Buch nicht nur die große Erfahrung, sondern auch die Begeisterung an, die sein Autorenteam für die Materie versprüht. Fast möchte man sich nochmal bei einem von innen in ein Praktikum setzen.

Die Abschnitte zur Isolierung, Kultivierung sowie Differenzierung von Bakterien und Pilzen sind rundum gelungen. So finden sich hier sehr ausführliche Anleitungen zur Isolierung verschiedener (auch anaerober) Bakteriengruppen aus Gewässer- und Bodenproben.
Gleiches gilt für das letzte Kapitel, das sich kompetent und informativ den lichtmikroskopischen Methoden widmet und auch die Theorie der einzelnen Lichtführungsverfahren liefert.

Leider ist dieses ansonsten überzeugende Machwerk nicht ohne Makel: Das vierte Kapitel zu den molekularbiologischen Methoden fällt qualitativ gegenüber den anderen Abschnitten ab und erscheint daher - auch aufgrund der Fülle entsprechender Literatur zu diesem Thema - überflüssig. An allen sich bietenden Stellen wird auf „kommerzielle Kits“ verwiesen. Das ist im Kontext großer Kurse bei stetig knapperen Budgets nicht nur fragwürdig, sondern auch didaktisch ärgerlich. Zudem sind die meisten Abschnitte in ihrer Knappheit sowohl praktisch als auch theoretisch kaum im Praktikumskontext anwendbar.

Was bleibt als Fazit? Zu fast 90 Prozent ist dieses Buch, das hoffentlich seinen Platz sowohl bei Betreuer/inne/ $\mathrm{n}$ als auch studentischen Teilnehmer/inne/n mikrobiologischer Grundkurse finden wird, eine Freude. Sie alle werden in inm eine reiche Quelle an Hintergrundinformationen und direkt anwendbaren Protokollen finden. Soll der molekularbiologische Teil beibehalten werden (was meiner Ansicht nach gar nicht nötig wäre), so würde ich mir hier eine Überarbeitung wünschen. Eine solche bleibt allerdings einer zweiten Auflage vorbehalten, die dieses auch hinsichtlich seines PreisLeistungs-Verhältnisses überzeugende Buch wirklich verdient hätte. In diesem Zusammenhang könnte man auch die wenigen Redundanzen bei den mikroskopischen Methoden zwischen den ersten und dem letzten Kapitel noch bereinigen.

Thorsten Mascher, TU Dresden, thorsten.mascher@tu-dresden.de

* Funding Open Access funding enabled and organized by Projekt DEAL.
* Open Access Dieser Artikel wird unter der Creative Commons Namensnennung 4.0 International Lizenz veröffentlicht, welche die Nutzung, Vervielfältigung, Bearbeitung Verbreitung und Wiederaabe in jeglichem Medium und Format erlaubt, sofern Sie den/die ursprünglichen Autor(en) und die Quelle ordnungsgemäß nennen, einen Link zur Creative Commons Lizenz beifügen und angeben, ob Änderungen vorgenommen wurden. Die in diesem Artikel enthaltenen Bilder und sonstiges Drittmaterial unterliegen ebenfalls der genannten Creative Commons Lizenz, sofern sich aus der Abbildungslegende nichts anderes ergibt. Sofern das betreffende Material nicht unter der genannten Creative Commons Lizenz steht und die betreffende Handlung nicht nach gesetzlichen Vorschriften erlaubt ist, ist für die oben aufgeführten Weiterverwendungen des Materials die Einwilligung des jeweiligen Rechteinhabers einzuholen. Weitere Details zur Lizenz entnehmen Sie bitte der Lizenzinformation auf http://creativecommons.org/ licenses/by/4.0/deed.de. 